\title{
OPTIMIZATION AND CHARACTERIZATION OF ION ACTIVATED OCULAR IN-SITU GEL FORMULATION FOR BACTERIAL CONJUNCTIVITIS
}

\author{
ANKITA KAPOOR*, G. D. GUPTA \\ ISF College of Pharmacy Moga, Affiliated to I. K. Gujral Punjab Technical University, Jalandhar \\ Email: ankita8620@yahoo.com
}

Received: 15 Apr 2020, Revised and Accepted: 29 May 2020

\begin{abstract}
Objective: The present research work aims at describing the formulation, optimization and evaluation of ion activated ocular in-situ gel of gatifloxacin for treatment of bacterial conjunctivitis so as to overcome patient inconvenience, precorneal drug elimination, variation in efficacy, vision blurring and frequent instillation associated with conventional eye drops and ointments.
\end{abstract}

Methods: In-situ gel was prepared using gellan gum as an ion activated phase transition polymer and HPMC K100M as release retardant. Gatifloxacin was characterized by spectrophotometry. Crystalline state of the drug was determined using X Ray Diffraction study. The developed formulation exhibited instantaneous gel formation in simulated lacrimal fluid ( $\mathrm{pH} 7.4$ ), which was further evaluated for its rheology, irritancy parameters, in vitro release, trans-corneal permeation and antimicrobial activity.

Results: Gatifloxacin exhibited $\lambda_{\max } 286 \mathrm{~nm}$ obeying Beer Lambert's law and pH-dependent solubility at a pH range of 2 to $4.0 .6 \%$ gellan gum and $0.4 \%$ HPMC K100M were optimized in the formulation which exhibited a viscosity of $55 \mathrm{cps}$ in sol form and $325 \mathrm{cps}$ in gel form with pseudoplastic behavior and prolonged in vitro release. Permeation of formulation was $75.8 \%$ in 7 h with log P of drug 0.59 . Developed isotonic and non-irritant formulation had a lower apparent permeability coefficient of $8.15 \times 10^{-5} \mathrm{~cm} / \mathrm{sec}$ as compared to marketed formulation.

Conclusion: A Formulation can be viewed as an efficacious medicine by virtue of its higher zone of inhibition, ability to enhance precorneal residence time and consequently ocular bioavailability with lesser frequency of administration attributed to slow and prolonged diffusion of the drug from the polymeric solutions.

Keywords: Gatifloxacin, In-situ, Pseudo plastic, Pre-corneal Residence, Isotonic, Rheology, On activated

(C) 2020 The Authors. Published by Innovare Academic Sciences Pvt Ltd. This is an open access article under the CC BY license (http://creativecommons.org/licenses/by/4.0/) DOI: http://dx.doi.org/10.22159/ijap.2020v12i4.37925. Journal homepage: https://innovareacademics.in/journals/index.php/ijap

\section{INTRODUCTION}

The term "conjunctivitis" encompasses a broad group of conditions referred as inflammation of the conjunctiva. The inflammation can be hyperacute, acute or chronic and infectious or noninfectious in origin. Conjunctivitis is the most common cause of red-eye. Most frequently, conjunctivitis (and thus red eye) is caused by bacterial or viral infection [1]. Bacterial conjunctivitis is usually caused by Staphylococcus aureus, Streptococcus pneumoniae, Haemophilus species, Pseudomonas aeruginosa, and Chlamydia trachomatis [2-4]. Topical administration of eye drops in the lower cul-de-sac is the most common method of drug delivery for the treatment of ocular disease [5]. However, one of the major problems encountered with the eye drops is the rapid and extensive elimination induced by tear turnover, blinking, and drainage of formulation, which leads to short precorneal residence time and poor ocular bioavailability. As a result, frequent instillation of eye drops is needed in order to achieve the desired drug concentration and therapeutic effect [6]. An increase in dosing frequency or use of the highly concentrated solution to compensate for short ocular residence time is undesirable because of poor patient compliance and risk of toxicity due to ophthalmic absorption via the nasolacrimal duct [7]. To increase ocular bioavailability and duration of drug action, various ophthalmic vehicles, i.e., viscous solutions [8], ointments/gels [9], and polymer inserts [10] have been used. These ocular drug delivery systems, however, have not been used extensively owing to some drawbacks, such as blurred vision from ointments, lack of patient compliance from inserts and sticking of eyelids from the gel.

An ideal ophthalmic dosage form is one that can sustain the drug release and remain in pre-corneal contact for an extended period of time. A significant increase in the residence time of the formulation and consequently, drug bioavailability can be achieved by delivery systems based on the concept of in-situ gelation [11]. These delivery systems consist of polymers that exhibit sol to gel phase transition due to changes in specific physiological conditions $\mathrm{pH}$, temperature and ionic strength) in the eye [12]. Depending upon the method employed to cause a phase transition on ocular surface, three types of systems are recognized, i.e., $\mathrm{pH}$ triggered systems-cellulose acetate hydrogen phthalate latex [13] and Carbopol [14-16], temperature-dependent systems-pluronic [17-19] and tetronics [20] and ion activated systems-gellan gum [21, 22] and sodium alginate [23]. Smart polymeric systems represent promising means of delivering the drugs; these polymers undergo sol-gel transition, once administered.

Gatifloxacin is a fourth-generation flouroquinolone antibiotic used for the treatment of bacterial conjunctivitis. It is commercially available in the form of an eye drops and ointment. The topical ophthalmic administration of $0.3 \%$ gatifloxacin solution is indicated in case of severe infection.

The objective of the present work involved the development of an in-situ gel formulation using an ion-activated phase transition polymer to effectively deliver the drug into the eye for sustained drug release and enhanced ocular drug bioavailability.

\section{MATERIALS AND METHODS}

\section{Materials}

Gatifloxacin was used as an active pharmaceutical ingredient and was procured as a gift sample from M/s. Alkem Laboratories Ltd (Mumbai, India). Gellan gum and HPMC were procured from Chempure (Mumbai, India) and Colorcon Asia Pvt. Ltd. (Mumbai, India), respectively. The nutrient agar medium was purchased from Hi-Media (India). Microbial strains were available at ISF Moga to perform the study, which was procured from MTCC Chandigarh. Whole eyeballs of goat were procured from the slaughter house. All the other reagents were used in the present study were of analytical grade.

\section{Preformulation studies}

Determination of $\lambda_{\max }$ by UV spectrophotometer

One hundred mg of gatifloxacin drug powder was accurately weighed and transferred to a $100 \mathrm{ml}$ volumetric flask. It was 
dissolved in an adequate amount of distilled water and the volume was made up to $100 \mathrm{ml}$ with distilled water so as to obtain a stock solution of $1000 \mu \mathrm{g} / \mathrm{ml}$ [24]. A dilution of $20 \mu \mathrm{g} / \mathrm{ml}$ concentration was made from the above stock solution with the distilled water and the resulting solution was scanned on a double-beam UV-visible spectrophotometer (Shimadzu 1700) between wavelength ranges of $200 \mathrm{~nm}$ to $400 \mathrm{~nm}$ [25].

\section{Infrared spectral analysis of gatifloxacin drug sample}

Gatifloxacin powder sample was compressed into a pellet al. ong with $\mathrm{KBr}$ (KBr pellet technique) using a shimadzu hydraulic press [26]. The FTIR spectrum of the drug was recorded between the wavenumber regions of $500-4000 \mathrm{~cm}^{-1}$ on an FTIR spectrophotometer (Shimadzu) [27].

\section{Melting point determination}

The melting point of gatifloxacin was determined using the open capillary method. The drug sample was filled into a capillary and placed in a Thiel's tube filled with liquid glycerol. The tube was heated and the temperature at which the drug melted was noted.

\section{$\mathrm{X}$-ray diffraction study of gatifloxacin drug sample}

X-ray diffraction analysis was performed using Rigaku diffractometer (high beam monochromatic) using $\mathrm{Cu} \mathrm{K} \alpha$ radiation, which was generated at $40 \mathrm{Kv}$ and $100 \mathrm{~mA}$ at $\lambda 1.5418 \AA \AA$. The data were collected over an angular range from $5-30^{\circ}$ in a continuous mode.

\section{Analytical method development}

\section{Preparation of calibration curve of gatifloxacin in distilled water}

Ten mg of gatifloxacin drug sample was accurately weighed and transferred into a $100 \mathrm{ml}$ volumetric flask. It was dissolved in an adequate amount of distilled water and the volume was made up to $100 \mathrm{ml}$ with distilled water to obtain a stock solution of $100 \mu \mathrm{g} / \mathrm{ml}$. Appropriate dilutions were made in distilled water from the above stock solution in the concentration range of $2-14 \mu \mathrm{g} / \mathrm{ml}$ and were analyzed spectrophotometrically at $286 \mathrm{~nm}$ against a blank prepared in the same manner. The absorbance data for different concentrations were subjected to regression analysis.

\section{Preparation of calibration curve of gatifloxacin in simulated lacrimal fluid}

Ten mg of gatifloxacin drug sample was accurately weighed and transferred into a $100 \mathrm{ml}$ volumetric flask. It was dissolved in an adequate amount of distilled water and the volume was made up to $100 \mathrm{ml}$ to obtain a stock solution of $100 \mu \mathrm{g} / \mathrm{ml}$. Appropriate dilutions were made in simulated lacrimal fluid from the above stock solution the concentration range of $2-14 \mu \mathrm{g} / \mathrm{ml}$ and were analyzed spectrophotometrically at $286 \mathrm{~nm}$ against a blank prepared in the same manner. The absorbance data for different concentrations were subjected to regression analysis.

\section{Partition coefficient $(\log P)$ determination}

The partition coefficient (P) is the quotient of two concentrations and is usually given in the form of its logarithm to base $10(\log \mathrm{P})$. Partition coefficient of the gatifloxacin drug sample was determined by saturating $10 \mathrm{ml}$ of octanol with $10 \mathrm{ml}$ of distilled water for $30 \mathrm{~min}$. Twenty mg of the drug was added to this blend and was moderately shaken for 1 hour with $5 \mathrm{~min}$ interval. The two solvent layers were separated through separating funnel and separately filtered through a Whatman ${ }^{\circledR}$ filter paper No 41 and the amount of gatifloxacin dissolved in each phase, was determined spectrophotometrically at $286 \mathrm{~nm}$ against reagent blank prepared in the same manner on a UV-visible spectrophotometer (Shimadzu 1700).

\section{Solubility studies}

The solubility of the gatifloxacin drug sample was determined in distilled water, buffer solutions of $\mathrm{pH} 1.2,2.0,4.0,6.0,7.4,1 \%$ Tween 80, disodium edetate, and simulated lacrimal fluid. An excess amount of drug was placed into suitable screw-cap vials containing
$10 \mathrm{ml}$ of each solvent, as mentioned above. The vials were properly sealed and vortexed for $10 \mathrm{~min}$. They were placed on the orbital flask shaker (Khera Instruments Pvt. Ltd. India) at room temperature for $24 \mathrm{~h}$. Then the samples were filtered, suitably diluted, and analyzed spectrophotometrically using a UV-visible spectrophotometer (Shimadzu 1700) at $286 \mathrm{~nm}$.

\section{Preparation of formulations}

Boric acid and disodium edetate were dissolved in distilled water. Gellan gum and HPMC were then dissolved in the above solution. The required quantity of gatifloxacin to give a final drug concentration of $0.3 \% \mathrm{w} / \mathrm{v}$ was added to the polymeric solution and stirred until dissolved and then phenylmercuric nitrate was added to it as a preservative. The formulations were filled amber colored glass vials, closed with rubber closures and sealed with aluminum caps. The formulations, in their final pack, were terminally sterilized by autoclaving at $121{ }^{\circ} \mathrm{C}$ temperature, 15 psi pressure for $15 \mathrm{~min}$. The sterilized formulations were stored in a refrigerator until further use.

\section{Gelation studies}

The gelation studies were carried out in cylindrical tubes, which were filled with $5 \mathrm{ml}$ of simulated lacrimal fluid (SLF). $50 \mu \mathrm{l}$ of the formulation containing ponceau red dye was added with a conventional dropper to a tube containing SLF and was then visually assessed for the gel formed and time for gelation as well as time is taken for the gel formed to dissolve.

\section{Evaluation of formulations}

\section{Physiochemical characterization}

The clarity of the formulation was evaluated by visual observation against white and black backgrounds for presence of any particulate matter. $\mathrm{pH}$ of the formulations was determined by $\mathrm{pH}$ meter (Cyberscan 510).

\section{Drug content uniformity}

Drug content analysis of prepared in-situ gelling systems was carried out using a spectrophotometric method. The assay of these formulations was carried out by diluting equivalent of $100 \mu \mathrm{l}$ of (Gatifloxacin) the formulation to $25 \mathrm{ml}$ with distilled water in the sterilized volumetric flask. The absorbance was estimated spectrophotometrically at $286 \mathrm{~nm}$ by using a double-beam UVvisible spectrophotometer (Shimadzu 1700).

\section{Viscosity and rheology}

The viscosity of the formulation was determined by Brookfield Viscometer (LVT model) [13]. To assess the gelation of formulation on instillation and mixing with lacrimal fluid, the viscosity measurements were also taken after diluting the formulation with the simulated lacrimal fluid (SLF). SLF comprised of 0.670 g sodium chloride, $0.200 \mathrm{~g}$ sodium bicarbonate and $0.008 \mathrm{~g}$ calcium chloride dihydrate and distilled water q. $\mathrm{s}$ to $100 \mathrm{~g}$, which simulated the cation content of lacrimal fluid $[19,31]$. Viscosity of the sample solution was measured over a range of 0.3 to $30 \mathrm{rpm}$ speed. The hierarchy of speed was reversed from 30 to $0.3 \mathrm{rpm}$. The average of the two dial readings was used to calculate the viscosity.

\section{Gel consistency test}

The firmness, consistency and cohesiveness of hydrogels are assessed using a texture analyzer, which mainly indicates the flow behavior of sol so the formulation can be easily administered in vivo. Higher values of adhesiveness of gels are needed to maintain intimate contact with surfaces like tissues [32]. Gel consistency was studied using Texture Analyzer TA-XT Plus (Stable Microsystems). Gelation of the formulation was induced by adding SLF to it. A test was carried out in a standard back extrusion rig using $50 \mathrm{~mm}$ (diameter) container filled approximately up to $75 \%$ with the formulation. An extrusion disc of $45 \mathrm{~mm}$ diameter was positioned centrally over the sample container. The probe used was calibrated for the force and distance measurement before use [33]. Positive area in the graph represents consistency and positive force represents firmness of sample. Negative area represents an index of viscosity and negative force represents cohesiveness. 


\section{Isotonicity evaluation}

Isotonicity, which is an important characteristic of the ophthalmic formulations, had to be maintained to prevent tissue damage and irritation to the eye. Smear of resuspended RBCs with gatifloxacin in-situ gel formulation was prepared and observed under the polarizing microscope (Leica) at 45x magnification [34, 35]. The same procedure was followed for the marketed gatifloxacin eye drops (Gatiquin ${ }^{\mathrm{TM}}$ ), isotonic solution (negative control) as well as hypertonic and hypotonic solution (positive controls). Size and shape of the RBCs with developed gatifloxacin in-situ gel formulation was compared to that with marketed gatifloxacin eye drops (Gatiquin ${ }^{\mathrm{TM}}$ ) as well as with the positive and negative controls.

\section{In vitro drug release study}

The in vitro drug release of the gatifloxacin in-situ gel formulation was estimated using modified USP dissolution apparatus-1 (Electrolab). Whatman ${ }^{\circledR}$ filter paper No 41 was placed in the USP basket. It was then wetted by dipping in a solution of simulated lacrimal fluid for one minute to ensure the intimate contact of the release medium with the formulation. Then $100 \mu \mathrm{l}$ of the formulation was applied to it. Fifty ml of simulated lacrimal fluid was filled in a beaker and the basket was rotated over its surface. A3-3 ml aliquots of samples were withdrawn at regular time intervals and replaced with an equal volume of fresh simulated lacrimal fluid. The samples were analyzed spectrophotometrically for gatifloxacin content using double beam UV-visible spectrophotometer (Shimadzu 1700) at $286 \mathrm{~nm}$.

\section{Kinetic modeling}

To study the release kinetics, data obtained from in vitro drug release studies were plotted as log cumulative percentage drug release versus log time to obtain a Korsmeyer Peppas plot. As the exponent $\mathrm{n}$ was 0.493 and $\mathrm{y}=0.493 \mathrm{x}+0.734 \mathrm{R}^{2}=0.989$, it represented non fickian diffusion [36, 37].

\section{In vitro trans corneal permeation study}

Whole eyeballs of goat were procured from slaughterhouse and safely transported to laboratory in cold condition. The corneas were carefully removed along with $5-6 \mathrm{~mm}$ of surrounding sclera and washed with cold saline solution. The washed corneas were stored in cold, freshly prepared solution simulated lacrimal fluid. Cornea was tied to one end of the hollow cylindrical glass tube and then its surface was wet by applying $14 \mu \mathrm{l}$ of simulated lacrimal fluid to simulate the physiological condition of the eyes. [19] $100 \mu \mathrm{l}$ of developed gatifloxacin in-situ gel formulation was spread over the corneal surface. The cylinder was then dipped into $50 \mathrm{ml}$ of SLF maintained at $37 \pm 0.5^{\square} \mathrm{C}$ so as to keep the corneal surface in contact with SLF stirred by rotation at 50 RPM. A 3-3 ml aliquots of fluid were withdrawn at fixed time intervals over a period of $7 \mathrm{~h}$ and was replaced with an equal volume of fresh SLF maintained at the same temperature. Aliquots of withdrawn samples were analyzed for drug content using a double beam UV-visible spectrophotometer (Shimadzu 1700) at $286 \mathrm{~nm}$.

\section{In vitro antimicrobial efficacy study}

In vitro antimicrobial efficacy study was carried out to ascertain the biological activity of ophthalmic sol-to-gel system against microorganisms. It was performed by an agar diffusion test using a cup-plate technique [19]. As gatifloxacin shows activity for both gram-positive and gram-negative microorganisms, Staphylococcus aureus (ATCC 25923) and Pseudomonas aeruginosa (ATCC27853) were used for the study. Strains were available at ISF Moga, which were procured from MTCC Chandigarh and are used for the present study. Developed gatifloxacin in-situ gel formulation, marketed eye drops of gatifloxacin (Gatiquin ${ }^{\mathrm{TM}}$ ) and placebo was poured in cups bored into the sterile nutrient agar previously seeded with Pseudomonas aeruginosa (ATCC 27853) and Staphylococcus aureus (ATCC 25923). After allowing diffusion of solution for $2 \mathrm{~h}$, the agar plates were incubated at $37 \pm 0.5{ }^{\circ} \mathrm{C}$ for $24 \mathrm{~h}$. The entire operation except the incubation was carried out under laminar airflow unit.

\section{Ocular irritation study (HET-CAM test)}

In developing a novel ophthalmic delivery system, an injury to the eye was taken into consideration. Since, eye being a sensitive, most delicate and yet most valuable of the sense organs, the injuries to the Cornea, Conjunctiva, and Iris were measured according to HET-CAM test (Hen's Egg Test-Chorio Allantoic Membrane). The potential ocular irritancy of a test substance was measured by its ability to induce toxicity in the Chorio Allantoic Membrane of a chick embryo [15, 23]. Fertilized hen's eggs weighing between 50-60 g were procured from a poultry farm. The eggs were then candled to discard the defective ones and were then incubated in a humidified incubator at $37{ }^{\circ} \mathrm{C}$ temperature and $75 \pm 5 \% \mathrm{RH}$. The trays containing eggs were rotated manually in a gentle manner every hour. After ninth days, a window $(2 \times 2 \mathrm{~cm})$ was cut on the pointed end of eggs through which $0.2 \mathrm{ml}$ of gatifloxacin in-situ gel formulation was instilled. A $0.9 \% \mathrm{NaCl}$ solution was used as a negative control because it is reported to be practically non-irritant being isotonic and physiologically compatible and $1 \%$ $\mathrm{NaOH}$ as a positive control in the present study. After instillation of the formulations, the scores were recorded [38, 39].

\section{RESULTS AND DISCUSSION}

\section{Preformulation studies}

\section{Determination of $\lambda_{\text {max }}$ By UV spectrophotometer}

The characterization of the gatifloxacin drug sample was done using spectrophotometric analysis. Gatifloxacin solution was scanned on a double-beam UV-visible spectrophotometer (Shimadzu 1700) between wavelength range of $200 \mathrm{~nm}$ to $400 \mathrm{~nm}$ as shown in fig. 1 and exhibited $\lambda_{\max } 286 \mathrm{~nm}$ which matches with the spectrum of gatifloxacin as reported in the literature $[24,25]$. So, this wavelength was used for the analysis of gatifloxacin solutions throughout the studies.

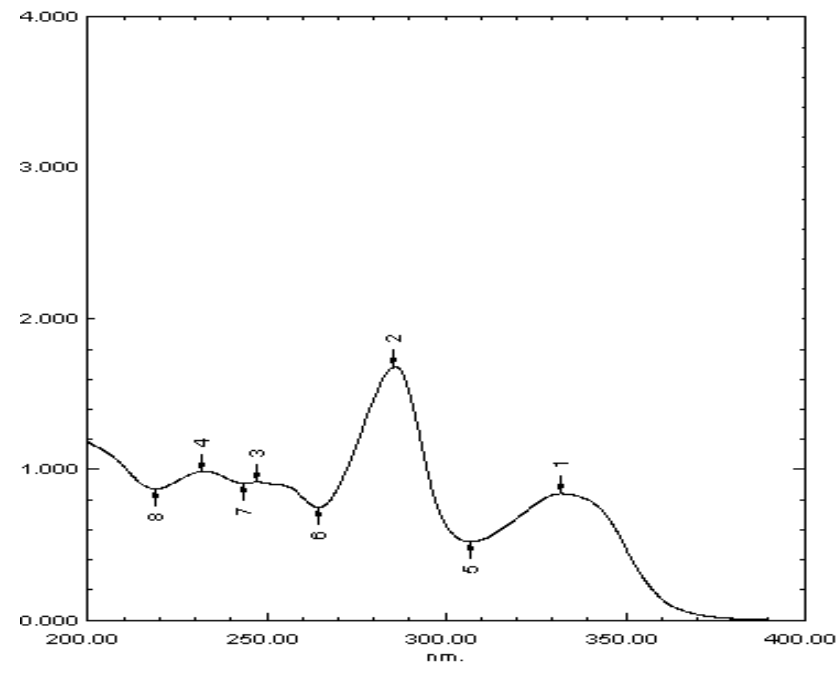

Fig. 1: UV spectrum of gatifloxacin drug sample in distilled water 


\section{Infrared spectral analysis of gatifloxacin drug sample}

Infrared spectrum of the gatifloxacin drug sample showed absorption bands for carbonyl group of quinone $\left(1631 \mathrm{~cm}^{-1}\right)$, methoxy group $\left(2802 \mathrm{~cm}^{-1}\right)$, unsaturation (3010 and $3076 \mathrm{~cm}^{-1}$ ), secondary amide $\left(3404 \mathrm{~cm}^{-1}\right)$, aromatic tertiary amine C-N stretch (1363 $\left.\mathrm{cm}^{-1}\right)$, and $\mathrm{Ar}-\mathrm{F}\left(1209 \mathrm{~cm}^{-1}\right)$ as represented in fig. 2 . It confirms the structure of drug as IUPAC name represents 1cyclopropyl-6-fluoro-1,4-dihydro-8-methoxy-7-(methylpiperazin-1yl)-4-oxo-3-qunolinecarboxylic acid sesquihydrate [26]. Additional fluorine atom is present at position 6 of the quinolone core, which affects the pharmacokinetic profile of fluoroquinolones in polar environments and improves their bioavailability and antibacterial activity.

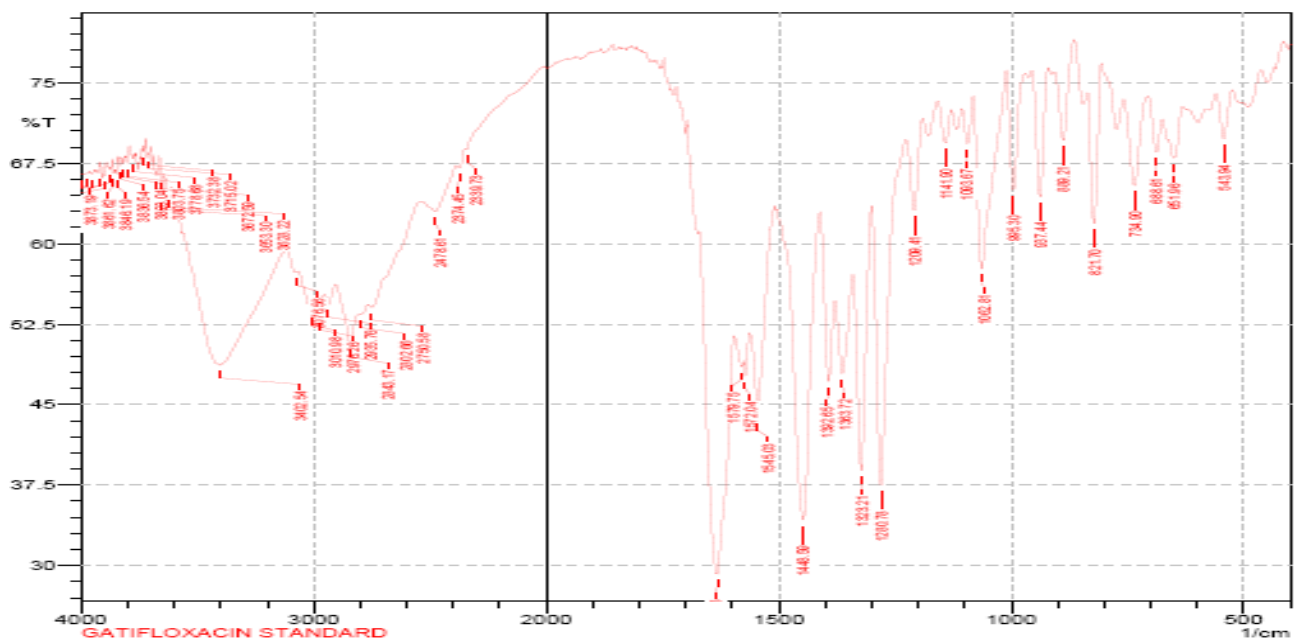

Fig. 2: FTIR spectrum of gatifloxacin drug sample

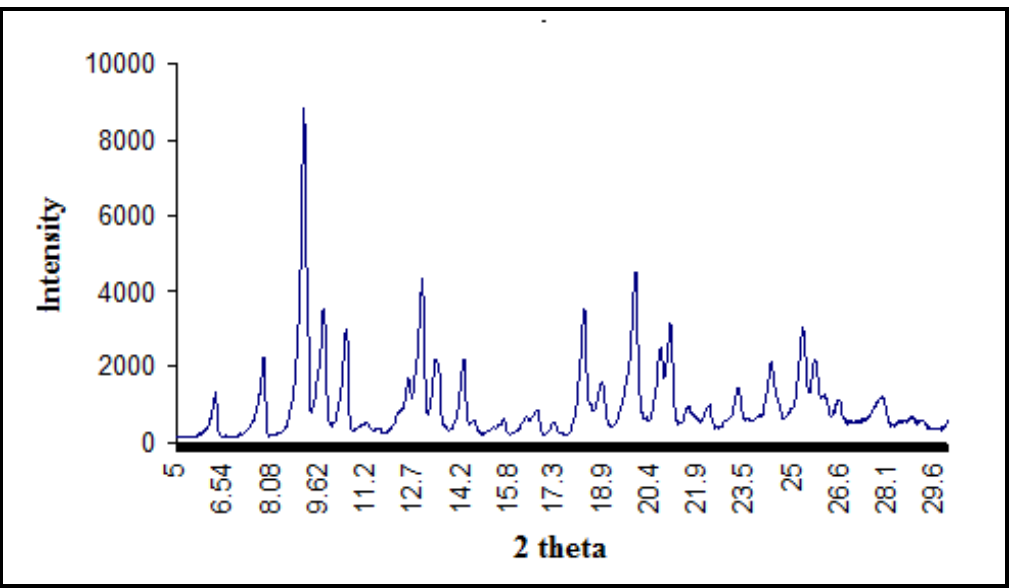

Fig. 3: X-ray diffractogram of gatifloxacin drug sample

\section{Melting point determination}

The melting point range of the gatifloxacin sample was founded around $180-183{ }^{\circ} \mathrm{C}$, which is very close to the theoretical melting point i.e., $182-185^{\circ} \mathrm{C}$ indicating gatifloxacin with high purity [26].

\section{$\mathrm{X}$-ray diffraction study of gatifloxacin drug sample}

The X-ray diffractograms in fig. 3 represents the values of scattering angles $(2 \theta)$, the inter planer $d$-spacings ( $d$-value), and the relative intensities $(I / I 0)$, which were automatically obtained on a digital printer and confirmed the crystalline structure of gatifloxacin drug sample [28]. It was inferred that the procured drug sample was of pure gatifloxacin sesquihydrate.

\section{Analytical method development}

Preparation of calibration curve of gatifloxacin in distilled water

Appropriate dilutions were made in distilled water in the concentration range of $2-14 \mu \mathrm{g} / \mathrm{ml}$ and were analyzed spectrophotometrically at $286 \mathrm{~nm}$ against a blank prepared in the same manner. The absorbance data for different concentrations were subjected to regression analysis. The observations are graphically represented in fig. 4. Equation of the line and R-square value was found to be. Conc. $=0.087 * \mathrm{~A}, \mathrm{R}=0.999$. The linearity of the calibration curves showed that Beer Lambert's law was obeyed in the concentration range of $4-14 \mu \mathrm{g} / \mathrm{ml}$ at $\lambda_{\max } 286 \mathrm{~nm}$

Preparation of calibration curve of gatifloxacin in simulated lacrimal fluid

Calibration curves of gatifloxacin in the simulated lacrimal fluid as represented in fig. 5 was prepared using a double beam UV-visible spectrophotometer (Shimadzu 1700). The linearity of the calibration curves showed that Beer Lambert's law was obeyed in the concentration range of $2-14 \mu \mathrm{g} / \mathrm{ml}$ at $\lambda_{\max } 286 \mathrm{~nm}$. Equation of the line and R-square value was found to be. Conc. $=0.097 * \mathrm{~A}, \mathrm{R}=0.999$. The linearity of the calibration curves showed that Beer Lambert's law was obeyed in the concentration range of $4-14 \mu \mathrm{g} / \mathrm{ml}$ at $\lambda_{\max } 286$ nm. 


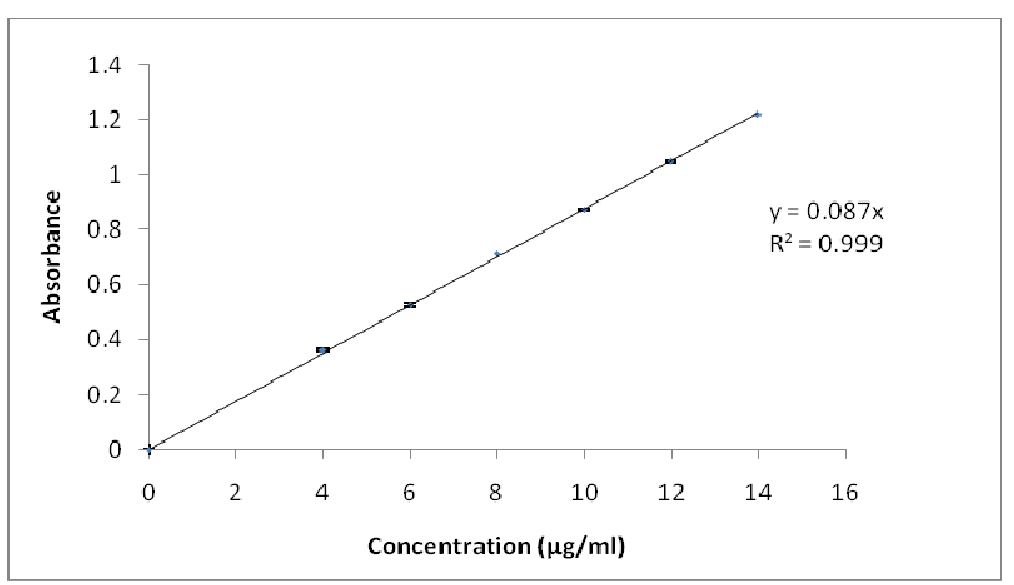

Fig. 4: Calibration curve of gatifloxacin in distilled water at $286 \mathrm{~nm}($ mean $\pm S D, n=3)$

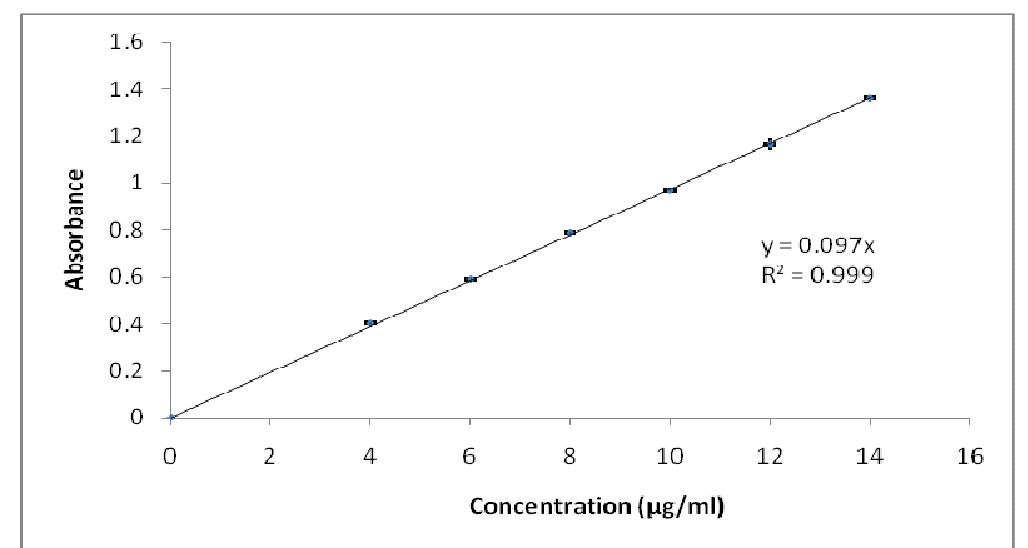

Fig. 5: Calibration curve of gatifloxacin in the simulated lacrimal fluid at $286 \mathrm{~nm}$ (mean $\pm S D, n=3$ )

Table 1: Solubility of gatifloxacin drug sample in different solvent media

\begin{tabular}{llll}
\hline S. No. & Solvent & Amount of drug dissolved (mg/ml) & Inference [30] \\
\hline 1 & Distilled water & $2.56 \pm 0.7$ & Slightly soluble \\
2 & Hydrochloric acid buffer (pH 1.2) & $64.3 \pm 1.2$ & Soluble \\
3 & Hydrochloric acid buffer (pH 2.0) & $56.8 \pm 0.9$ & Soluble \\
4 & Acetate buffer (pH 4.0) & $44.4 \pm 0.9$ & Soluble \\
5 & Phosphate buffer (pH 6.0) & $7.6 \pm 0.5$ & Slightly soluble \\
6 & Phosphate buffer (pH 7.4) & $3.73 \pm 0.1$ & Slightly soluble \\
7. & Simulated lacrimal fluid & $3.81 \pm 0.2$ & Slightly soluble \\
\hline
\end{tabular}

The values are expressed as mean $\pm \mathrm{SD}=\mathrm{Standard}$ Deviation from the mean, $\mathrm{n}=3$

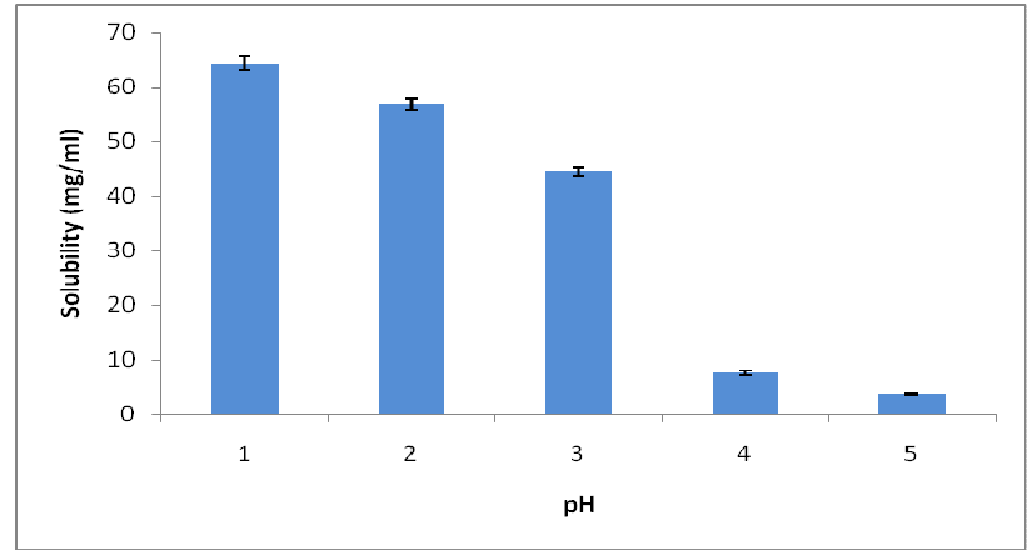

Fig. 6: $\mathrm{pH}$ dependent solubility profile of gatifloxacin drug sample (mean $\pm \mathrm{SD}, \mathrm{n}=3$ ) 


\section{Partition coefficient $(\log P)$ determination}

The Partition coefficient was calculated as the ratio of concentration of the drug in octanol to the concentration of the drug in water and then its logarithm was taken. The octanol-water partition coefficient ( $\log$ P) of drug sample was found to be 0.59 , which was similar to the calculated value from its structure, i.e., $\mathrm{ClogP} 0.6$ and also matches with that reported in the literature [29].

\section{Solubility studies}

It was also found that gatifloxacin having a crystalline structure exhibit pH-dependent solubility with an aqueous solubility of 40-60 $\mathrm{mg} / \mathrm{ml}$ at a $\mathrm{pH}$ range of 2 to 4 as represented in table 1 and fig. 6 .

\section{Preparation and optimization of formulation}

Composition of in-situ gelling formulations is represented in table 2 . Gellan gum was selected as an ion activated phase transition polymer for gel formation in-situ. Different concentrations of gellan gum, i.e., $0.1-0.7 \%$ were evaluated for the gelling property in physiological conditions. Out of which, only gellan gum solution of $0.6 \%$ exhibited desirable flow characteristics and resulted in instantaneous gelation in simulated lacrimal fluid, which was retained for an extended period of time as shown in table 3. Buffer solutions were earlier tried to impart the buffering capacity in the formulation, but that was found to result into gel formation due to probable interaction between ions and gellan gum. As isotonicity is a desirable attribute of an ophthalmic formulation, sodium chloride and boric acid were studied as an isotonicity adjusting agents. Since, gel formation was observed in the formulation containing sodium chloride hence boric acid was selected as an isotonicity adjusting agent. Phenyl mercuric nitrate was used as a preservative in the formulation. A $0.05 \%$ of disodium edetate was also added to enhance the solubility of gatifloxacin in water and prevent its crystallization in freeze-thaw conditions. HPMC K100M was incorporated as a release retardant in the formulation. The formulations, in their final pack were terminally sterilized by autoclaving at $121{ }^{\circ} \mathrm{C}$ temperature, 15 psi pressure for $15 \mathrm{~min}$. The sterilized formulations were stored in a refrigerator until further use. Composition of formulations is mentioned as below in table 2 .

Table 2: Composition of prepared in-situ gelling formulations

\begin{tabular}{lllll}
\hline Ingredients & Amount (g) & & & \\
\cline { 2 - 5 } & F 1 & F 2 & F 3 & F 4 \\
\hline Gatifloxacin & 0.3 & 0.3 & 0.3 & 0.3 \\
Gellan gum & 0.6 & 0.6 & 0.6 & 0.6 \\
Boric acid & 1.68 & 1.68 & 1.68 & 0.3 \\
Phenylmercuric nitrate & 0.002 & 0.002 & 0.002 & 0.6 \\
Disodium edetate & 0.05 & 0.05 & 0.05 & 0.002 \\
HPMC K100M & - & 0.3 & 0.4 & 0.05 \\
Distilled water & 100 & 100 & 0.5 & 0.5 \\
\hline
\end{tabular}

\section{Gelation studies}

Different concentrations of gellan gum, i.e., $0.1-0.7 \%$ were evaluated for the gelling property in physiological conditions. Out of which, only gellan gum solution of $0.6 \%$ exhibited desirable flow characteristics and resulted in instantaneous gelation in the simulated lacrimal fluid, which was retained for an extended period of time. The grading of gelling capacity is based up on the time taken to form the gel and time taken to dissolve the gel in simulated tear fluid. Formulations with good gelling capacity exhibit that gelation remains for longer periods once it occurs.

Table 3: Gel formation of Gellan Gum with simulated lacrimal fluid (SLF)

\begin{tabular}{ll}
\hline Concentration of gellan gum & Gelling property in SLF \\
\hline $0.1 \%$ & no gelation \\
$0.2 \%$ & no gelation \\
$0.3 \%$ & immediate gelation for few minutes \\
$0.4 \%$ & immediate gelation for few minutes \\
$0.5 \%$ & immediate gelation for few minutes \\
$0.6 \%$ & immediate gelation which last longer \\
$0.7 \%$ & immediate gelation which last longer \\
\hline
\end{tabular}

Table 4: Drug content uniformity

\begin{tabular}{ll}
\hline Formulation code & Drug content $\mathbf{( \% \pm S D )}$ \\
\hline F 1 & $99.3 \pm 0.45$ \\
F 2 & $97.8 \pm 0.11$ \\
F 3 & $98.7 \pm 0.75$ \\
F 4 & $96.0 \pm 0.60$ \\
F 5 & $97.1 \pm 0.05$ \\
\hline
\end{tabular}

The values are expressed as mean \pm SD Standard Deviation from the mean, $n=3$

\section{Evaluation of formulations}

\section{Physiochemical characterization}

The designed formulation was found to be clear by visual examination against white and black backgrounds. The $\mathrm{pH}$ of the formulation was determined to be between 6.2-6.3 by using $\mathrm{pH}$ meter (Cyberscan ${ }^{\circledR}$ 510). Formulations resulted in gel formation in SLF, clearly indicating phase transition behavior in the physiological conditions of the eye.

\section{Drug content uniformity}

Drug content uniformity was found to be between $98.7 \pm 0.75$ to $99.6 \pm 0.84 \%$ (Shimadzu 1700) at $286 \mathrm{~nm}$ as shown in table 4 .

\section{Viscosity and rheology}

Viscosity of the formulation was determined by Brookfield viscometer (LVT). The formulation exhibited a viscosity of $55 \mathrm{cps}$ in solution form and $325 \mathrm{cps}$ in gel form at $12 \mathrm{rpm}$. There were 6 folds 
increase in the viscosity of gel as compared to its sol form. It exhibited pseudoplastic behavior as there was shear thinning with the increase of angular velocity.

\section{Gel consistency test}

The developed formulation was further subjected to gel consistency studies on Texture Analyzer (TA-XT Plus). The consistency, firmness and cohesiveness of in-situ gelling system are assessed by using this device. Higher values of adhesiveness of gels are needed to maintain intimate contact with the mucus surface. Gel formed in simulated lacrimal fluid exhibited a consistency of $216.59 \mathrm{gm}$. sec, firmness $28.344 \mathrm{gm}$, index of viscosity $37.465 \mathrm{gm}$. sec, and cohesiveness$17.896 \mathrm{gm}$ as shown in table 5 and fig. 7 . The purpose of texture analyses was to provide information about the mechanical properties of samples. These properties can be directly correlated with sensory parameters in vivo and, therefore, are valuable in the development of products with desirable attributes that contribute to patient acceptability and compliance.

Table 5: Results of gel consistency test of developed gatifloxacin in-situ gel formulation

\begin{tabular}{llll}
\hline Area F-T 1:2 gm. sec & Area F-T 3:4 gm. sec & Force 1 gm & Force 2 gm \\
\hline 216.592 & -37.465 & 28.344 & -17.896 \\
\hline
\end{tabular}

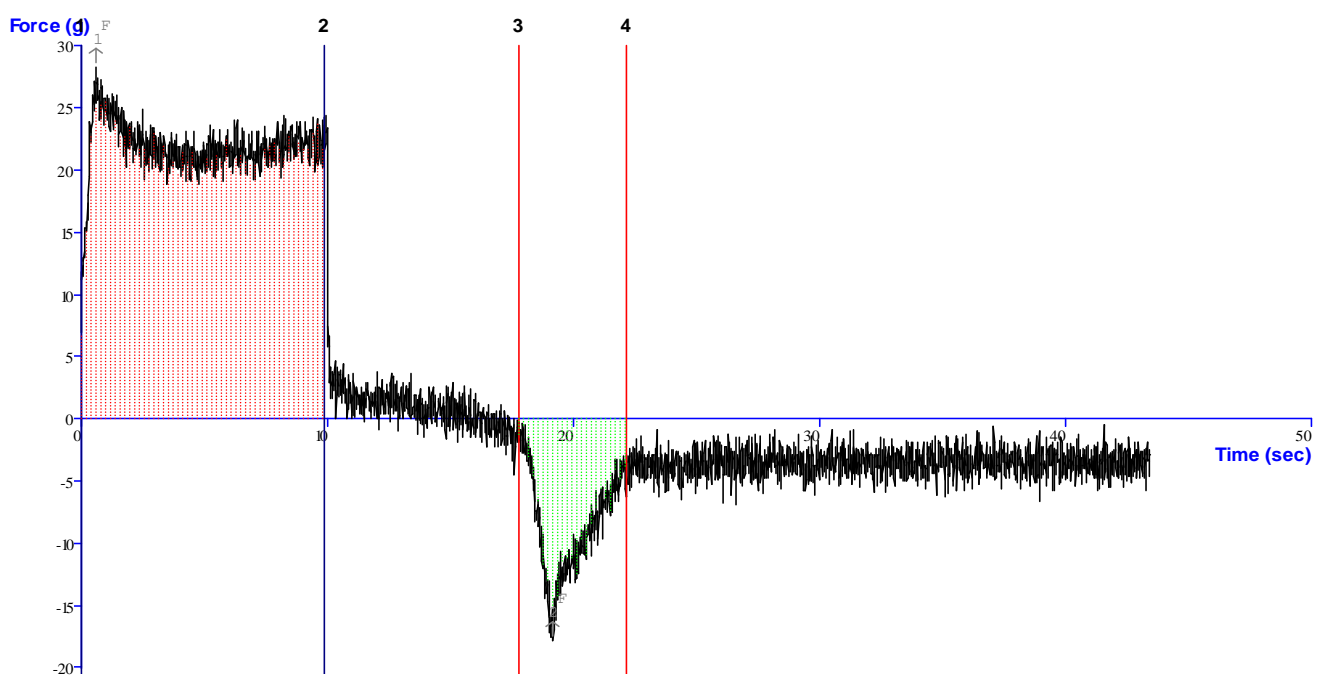

Fig. 7: Gel consistency test of developed gatifloxacin in-situ gel formulation

\section{Isotonicity evaluation}

Gatifloxacin in-situ gel formulation was found to be isotonic, as it exhibited no change in the size and shape of RBCs. The formulation was evaluated for the isotonicity and was also compared with the marketed eye drops, isotonic solution (negative control), hypertonic and hypotonic solution (positive controls). There was no change in the shape of the
RBCs after the addition of developed gatifloxacin in-situ gel formulation, while hypertonic solution resulted in shrinkage of the cells and hypotonic solution caused the bursting of the cells.

Hence, it was confirmed that the formulation was isotonic to the eyes. It was also compared with that of marketed gatifloxacin eye drops (Gatiquin $^{\mathrm{TM}}$ ) as shown in fig. 8.

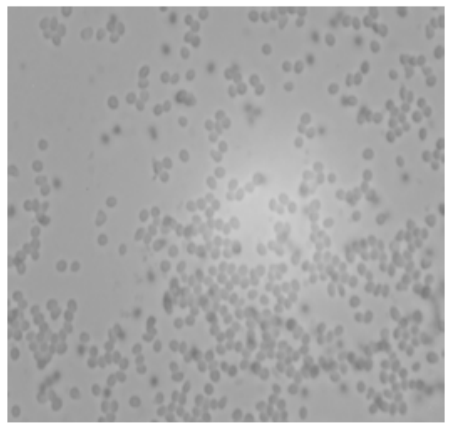

(a)

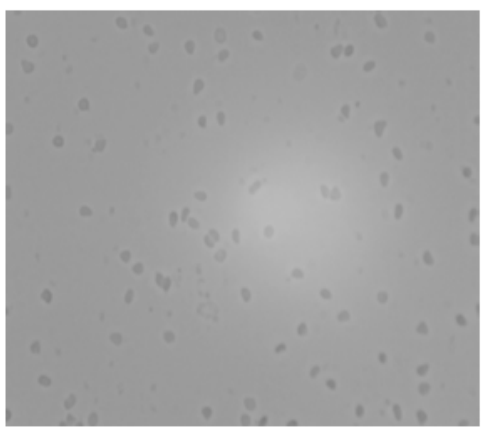

(b)

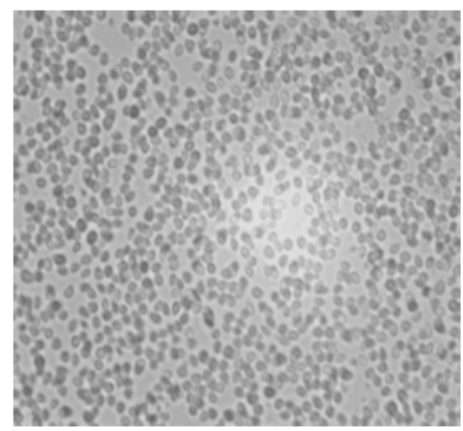

(c)

Fig. 8: (a) RBCs with developed in-situ gel formulation of gatifloxacin F3, (b) RBCs with marketed gatifloxacin eye drops (Gatiquin ${ }^{\mathrm{TM}}$ ), (c) RBCs with isotonic

\section{In vitro drug release study}

Combination of $0.6 \%$ gellan gum and $0.4 \%$ HPMC was selected, as it had satisfactory attributes of in-situ gelling property, flow characteristics and prolonged in vitro release over the duration of 8 h with $90.6 \%$ release as shown in fig. 9 .

\section{Kinetic modeling}

Data obtained from in vitro drug release studies were plotted as log cumulative percentage drug release versus log time, which is Korsmeyer Peppas plot $[36,37]$ as shown in fig. 10 . As the exponent $n$ was 0.493 , it it followed the anomalous transport. The drug release pattern obtained for 
the gelled samples as represented in fig. 9 and fig. 10 are characteristic for hydrophilic matrices. It is rapid in the beginning and proceeds at a rate that declined with time. When the formulation comes in contact with the simulated lacrimal fluid and gelation occurs, a pre-hydrated matrix is formed in which hydration and water penetration no longer limit drug release, leading to an apparent diffusion-controlled release. As gellan gum is a biodegradable polymer, it followed diffusion and matrix erosion of HPMC occurred due to hydrolytic cleavage of polymer chains and the result of kinetic data revealed that gatifloxacin followed zeroorder release kinetics independent of time.

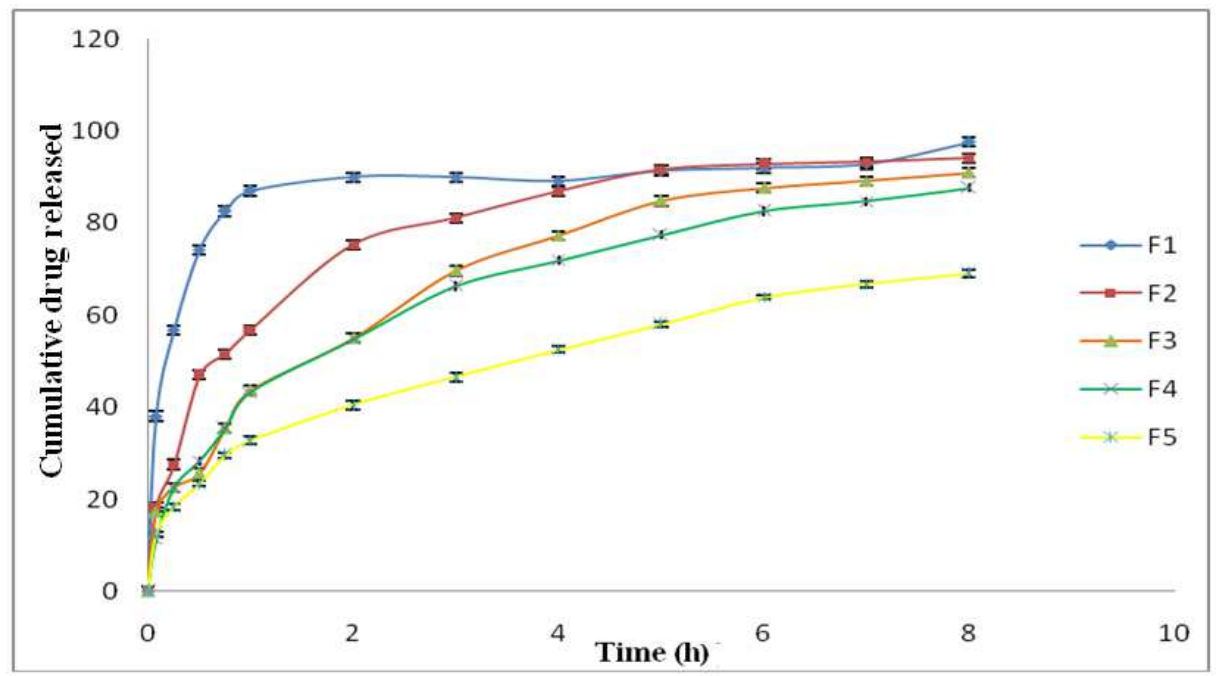

Fig. 9: In vitro drug release profile of gatifloxacin in-situ gel formulation in simulated lacrimal fluid (SLF) (mean \pm SD, $n=6)$

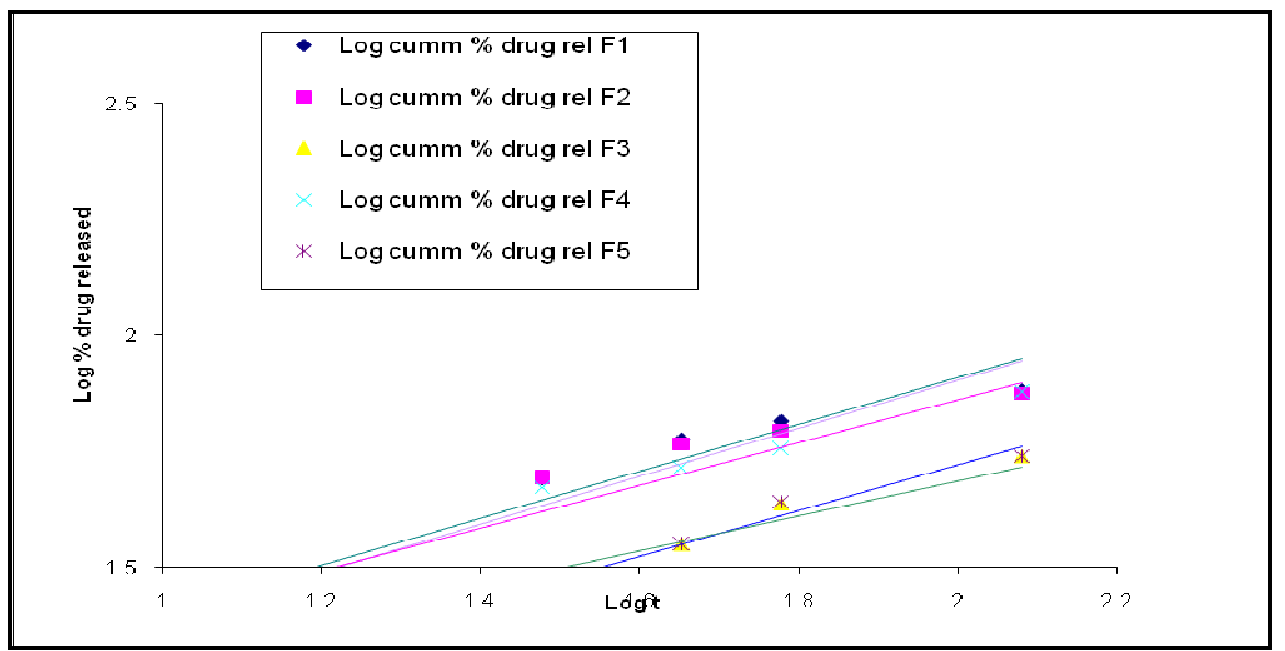

Fig. 10: Representation of korsmeyer peppas plot (mean $\pm S D, n=6$ )

\section{In vitro trans corneal permeation study}

Tran's corneal drug permeation study for gatifloxacin in-situ gel formulation was performed in the SLF (pH 7.4 and $\left.37 \pm 0.5^{\circ} \mathrm{C}\right)$ which exhibited the delayed release of drug from the polymeric matrix. The in vitro trans corneal permeation study across excised goat's cornea exhibited $74.8 \%$ drug permeability in $7 \mathrm{~h}$ with an apparent permeability coefficient of $8.25 \times 10^{-5} \mathrm{~cm} / \mathrm{sec}$. Prolonged precorneal retention in the conjunctival sac and slow drug release from the formulation could impart improved in vivo ocular bioavailability.

\section{In vitro antimicrobial efficacy study}

Diameter of the zone of inhibition observed with the developed formulation was higher as shown in fig. 11 than that of marketed preparation Gatiquin TM. Overall values of the diameter of the zone of inhibition against $P$. aeruginosa $(6.6 \mathrm{~cm})$ were higher than that of $S$. aureus $(4.3 \mathrm{~cm})$. The highest values obtained for the developed formulation in comparison to the marketed eye drops, which was 5.3 $\mathrm{cm}$ for $P$. aeruginosa and $4 \mathrm{~cm}$ for $S$. aureus could be attributed to slow and prolonged diffusion of the drug from the polymeric solutions.

\section{Ocular irritation study (HET-CAM test)}

Developed formulation was also found to be non-irritant to eyes. It was tested for irritation on the Chorio Allantoic Membrane of the chick embryo, which is a complete tissue, including veins, arteries and capillaries and responds to injury with a complete inflammatory process, a process similar to that induced in the conjunctival tissue of rabbit eyes [15, 23]. Each CAM was observed for 5 min after instillation for hemorrhage, coagulation and vessel lysis [40, 41]. Gatifloxacin in-situ gel formulation was found to be non-irritant to eyes exhibiting mean score of zero in HET-CAM test for ocular irritancy for $5 \mathrm{~min}$ as shown in table 6 . Since the immune response generated by chorioallantoic membrane of chicken simulates the ocular immune response of the human eye, the developed formulation can be presumed to be non-irritant to the eyes. 


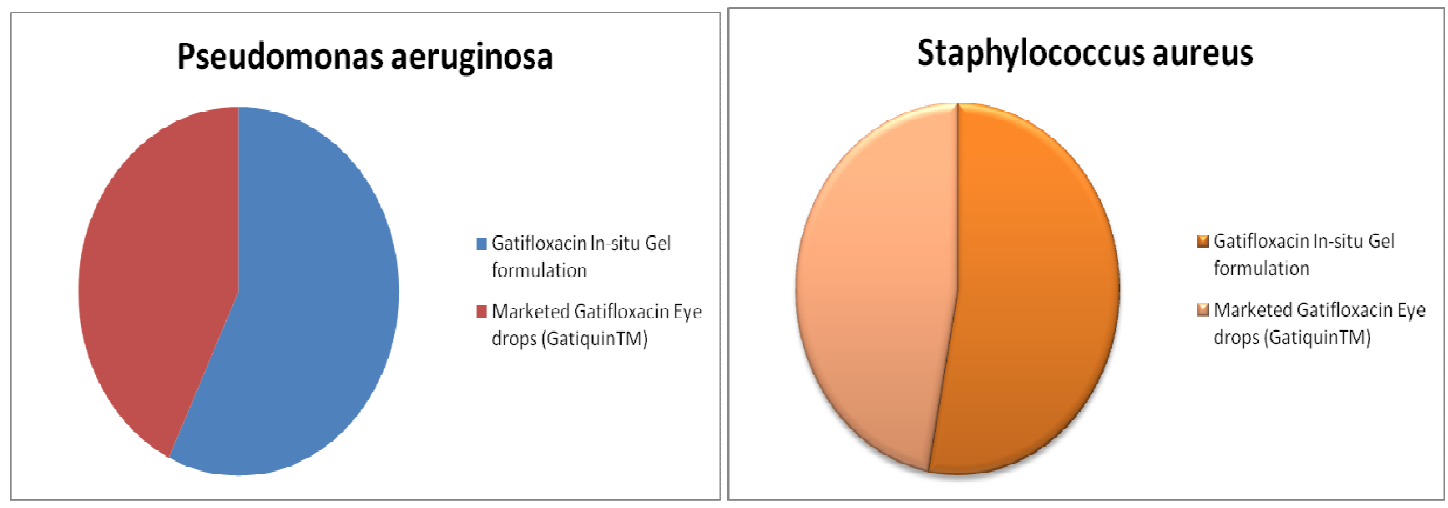

Fig. 11: Zone of inhibition exhibited by the developed gatifloxacin in-situ gel formulation F3 and marketed eye drop (Gatiquin ${ }^{\mathrm{TM}}$ )

Table 6: Observations for HET-CAM test

\begin{tabular}{lll}
\hline Test substance & Score & Inference \\
\hline $0.9 \% \mathrm{NaCl}$ & 0 & Non-irritant \\
Developed Formulation & 0 & Non-irritant \\
$1 \% \mathrm{NaOH}$ & 14.27 & Severe irritant \\
\hline
\end{tabular}

\section{CONCLUSION}

On the basis of observation made and results obtained, it can be concluded that the developed gatifloxacin in-situ gel formulation can overcome the drawbacks of the conventional ocular dosage forms. The developed formulation provided efficient therapy through the prolonged drug release of the drug over an $8 \mathrm{~h}$ period in vitro. A formulation could be easily removed from the package, presented a good spreadability on the corneal surface and adhere to the mucous layer without disintegrating, in order to prolong retention time. It exhibited better antimicrobial efficacy when compared with the marketed eye drops. The formulation was isotonic and devoid of any irritant effect to the eyes. The ease of administration, along with its ability to provide sustained release could result in a decrease in the frequency of administration thus enhancing patient compliance.

\section{ABBREVIATION}

SLF-Simulated Lacrimal Fluid, HET-CAM-Hens Egg test-Chorio Allantoic Membrane, HPMC-Hydroxy Propyl Methyl Cellulose, UVUltra Violet, RBC-Red Blood Cells, MTCC-Microbial Type Culture Collection and Gene Bank

\section{ACKNOWLEDGMENT}

Authors are thankful to I. K. Gujral Punjab Technical University, Jalandhar for academic support.

\section{FUNDING}

Nil

\section{AUTHORS CONTRIBUTIONS}

All the authors have contributed equally.

\section{CONFLICT OF INTERESTS}

The authors reported no conflict of interest. The authors alone are responsible for the content and writing of the paper.

\section{REFERENCES}

1. Morrow GL, Richard L. Conjunctivitis. Am Fam Physician 1998;57 Suppl 4:735-46.

2. Leung AKC, Hon KL, Wong AHC, Wong AS. Bacterial conjunctivitis in childhood: etiology, clinical manifestations, diagnosis, and management. Recent Pat Inflamm Allergy Drug Discovery 2018;12 Suppl 2:120-7.

3. Pichichero ME. Bacterial conjunctivitis in children: antibacterial treatment options in an era of increasing drug resistance. Clin Pediatr (Phila) 2011;50 Suppl 1:7-13.
4. Epling J, Smucny J. Bacterial conjunctivitis. Clin Evid 2005;2 Suppl 14:756-61.

5. Kristiina J, Tomi J, Urtii A. Ocular absorption following drug delivery. Adv Drug Delivery Rev 1995;16:3-19.

6. Sreeraj M, Mitra AK, Hughes PM. In ophthalmic drug delivery systems. Marcel Deckker, Inc., Kanas city, Missouri, U. S. A.; 2003. p. 1-5.

7. Balasubramaniam J, Pandit JK. Ion-activated in-situ gelling systems for sustained ophthalmic delivery of ciprofloxacin hydrochloride. Drug Delivery 2003;10:185-91.

8. Schoenwald RD, Ward RL, Desantis LM, Rochrs RE. Influence of high viscosity vehicles on miotic effect of pilocarpine. J Pharm Sci 1978;67:1280-4.

9. Maurice DM, Srinivas SD. Use of flourimetry in assessing the efficacy of a cation sensitive gel as an ophthalmic vehicle; comparison with scintigraphy. J Pharm Sci 1992;81:615-9.

10. Mishra DN, Gilhotra RM. Design and characterization of bioadhesive in-situ gelling ocular inserts of gatifloxacin sesquihydrate. DARU 2008;16:1-7.

11. Nanjawade BK, Manvi FV, Manjappa AS. In-situ-forming hydrogels for sustained ophthalmic drug delivery. J Controlled Release 2007;122:119-34.

12. Bolanle L. Development of a cysteamine in-situ gelling system for the treatment of corneal crystals in cystinosis. University of London, London; 2008.

13. Gurny R. Preliminary studies of porlonged acting drug delivery system for the treatment of glaucoma. Pharm Acta Helv 1981;56:130-2.

14. Naseem AC, Kohli K, Ali A. Preparation of in-situ froming ophthalmic gels of ciprofloxacin hydrochloride for the treatment of bacterial conjunctivitis: in vitro and in vivo studies. J Pharm Sci 2002;92:407-13.

15. Sultana Y, Aquil M, Ali D, Zafar S. Evaluation of carbopol-methyl cellulose-based sustained-release ocular delivery system for pefloxacin mesylate using rabbit eye model. Pharm Dev Technol 2006;11:313-9.

16. Srividya B, Rita M, Cardoza RM, Amin PD. Sustained ophthalmic delivery of ofloxacin from a pH triggered in-situ gelling system. J Controlled Release 2001;73:205-11.

17. Mansour M, Mansour S, Mortada ND, Abd Elhady SS. Ocular poloxamer-based ciprofloxacin hydrochloride in-situ forming gels. Drug Dev Ind Pharm 2008;34:744-52.

18. Ma WD, Xu H, Wang C, Nie SF, Pan WS. Pluronic F127-gpoly(acrylic acid) copolymers as in-situ gelling vehicle for ophthalmic drug delivery system. Int J Pharm 2008;350:247-56.

19. Gupta H, Jain S, Mathur R, Mishra P, Mishra AK, Velpandian T. Sustained ocular drug delivery from temperature and $\mathrm{pH}$ triggered novel in-situ gel system. Drug Delivery 2007;14:507-15. 
20. Vadnere M, Amidon G, Lendenbaum S, Haslam JL. Thermodynamic study on the gel-sol transition of some pluronic polyols. Int J Pharm 1984;22:207-18.

21. John C, Katarina E, Roger P, Katarina J. Rheological evaluation of Gelrite ${ }^{\circledR}$ in-situ gels for ophthalmic use. Eur J Pharm Sci 1998;6:113-9.

22. Balasubramaniam J, Pandit JK. Ion-activated in-situ gelling systems for sustained ophthalmic delivery of ciprofloxacin hydrochloride. Drug Delivery 2003;10:185-91.

23. Smadar C, Esther L, Amira T, Yael P. A novel in-situ-forming ophthalmic drug delivery system from alginates undergoing gelation in the eye. J Controlled Release 1997;44:201-8.

24. Venugopal K, Saha RN. New, simple and validated UVspectrophotometric methods for the estimation of gatifloxacin in bulk and formulations. Farmaco 2005;60 Suppl 11-12:906-12.

25. Doijad RC, Manvi FV, Malleswara VSN, Aalse P. Sustained ophthalmic delivery of gatifloxacin from in-situ gelling system. Indian J Pharm Sci 2006;68 Suppl 6:814-8.

26. Indian Pharmacopoeia. The Indian Pharmacopoeia Commission, Govt of India. Ministry of health and family Welfare: Ghaziabad; 2007. p. 540.

27. Kalam MA, Sultana Y, Samad A, Ali A, Aquil M, Sharma M, et al. Gelrite based in vitro gelation ophthalmic drug delivery system of gatifloxacin. J Dispersion Sci Technol 2008;29:89-96.

28. Valerie N, Shlomit W, Greta S. Crystalline forms of gatifloxacin. US Patent 7,396,839 B2; 2008.

29. Boubarkar BB, Corinne A, Celine V, Arnaud C, Claudine Q. Activity of gatifloxacin in an in vitro pharmacokineticpharmacodynamic model against Staphylococcus aureus strains either susceptible to ciprofloxacin or exhibiting various levels and mechanisms of ciprofloxacin resistance. Antimicrob Agents Chemother 2006;50 Suppl 6:1931-6.

30. United States Pharmacopoeia-24 NF-19. The United State Pharmacopoeial Convention Incorporation: Rockville, M. D.; 2000. p. 10.
31. Kashyap N, Viswanad B, Sharma G, Bhardwaj V, Ramarao P, Kumar MNV. Design and evaluation of biodegradable, bio sensitive in-situ gelling systems for pulsatile delivery of insulin. Biomaterials 2007;28:2051-60.

32. Kurniawansyah IS, Sopyan I, Wathoni N, Fillah Dl, Praditya RU. Application and characterization of in-situ gel. Int J Appl Pharm 2018;10 Suppl 6:34-7.

33. Hurler J, Engesland A, Poorahmary B, Natasa K, Basnet S. Improved texture analysis of Hydrogel characterization: gel cohesiveness, adhesiveness and hardness. J Appl Polym Sci 2012;125 Suppl 1:180-8.

34. Gokulgandhi MR, Parikh JR, Barot M, Modi DM. A pH triggered in-situ gel forming ophthalmic drug delivery system for tropicamide. Drug Delivery Technol 2007;5:44-9.

35. Hiremath SSP, Dasankoppa FS, Nadaf A, Jamakandi VG. Formulation and evaluation of a novel in-situ gum-based ophthalmic drug delivery system of linezolid. Sci Pharm 2008;76:515-32.

36. Korsmeyer RW, Gurny R, Doelker E, Buri P, Peppas NA. Mechanisms of solute release from porous hydrophilic polymers. Int J Pharm 1983;15:25-35.

37. Peppas NA. Analysis of Fickian and non-fickian drug release from polymers. Pharm Acta Helv 1985;60:110-1.

38. Laddha UD, Mahajan HS. An insight to ocular in-situ gelling systems. Int J Appl Pharm 2017;6 Suppl 2:31-40.

39. Rupenthal ID. Ocular delivery of antisense oligonucleotides using colloidal carriers: improving the wound repair after corneal surgery. PhD Thesis The University of Auckland, NewZealand.20; 2008.

40. Dabir PD, Shahi SR, Deore SV. Opthalmic in situ gel: a review. Eur J Pharm Med Res 2016;3:205-15.

41. Vinardell MP, Mitjans M. Alternative methods for eye and skin irritation tests: an overview. J Pharm Sci 2008;97:46-59. 